\title{
Undergraduate Research Opportunities via Google
}

\author{
C.C. Chancey, Editor \\ American Journal of Undergraduate Research \\ University of Northern lowa \\ Cedar Falls, lowa 50614-0150 USA
}

This issue represents the beginning of AJUR's third year of activity. To the extent that research paper submissions to this journal measure the health of the undergraduate research endeavor, undergraduate research is thriving in many parts of the world. But the field, of course, is not level.

The following list is the result of a simple and quick test. The text strings "undergraduate research" + "[country]" were entered into a Google web search [http://www.google.com/]. The number of resulting "hits" is given below.

Needles to say, this is not a good scientific measure: all country names were in their English forms, the search assumed English text, and the search results surely included some hits that are not necessarily measures of undergraduate research activity in a country [e.g., an Oregon State University "undergraduate research" program in "Honduras" tells us more about Oregon State than it does about Honduras]. But, these simple results are surely a partial measure of research opportunities for undergraduates in those countries.

\begin{tabular}{|l|c|}
\hline \multicolumn{1}{|c|}{ Country } & Google "Hits" \\
\hline United States & 191,000 \\
\hline Canada & 25,200 \\
\hline United Kingdom & 23,500 \\
\hline Germany & 16,400 \\
\hline Japan & 16,200 \\
\hline France & 14,800 \\
\hline China & 14,300 \\
\hline Mexico & 12,500 \\
\hline Australia & 12,400 \\
\hline India & 9,940 \\
\hline Italy & 9,900 \\
\hline Spain & 8,480 \\
\hline
\end{tabular}

\begin{tabular}{|l|c|}
\hline Russia & 7,110 \\
\hline Brazil & 5,850 \\
\hline Korea & 5,760 \\
\hline Switzerland & 5,440 \\
\hline South Africa & 5,100 \\
\hline New Zealand & 5,020 \\
\hline Sweden & 4,830 \\
\hline Turkey & 4,690 \\
\hline Egypt & 3,870 \\
\hline Denmark & 3,470 \\
\hline Chile & 3,380 \\
\hline Argentina & 3,110 \\
\hline Norway & 2,790 \\
\hline Nigeria & 2,020 \\
\hline Pakistan & 2,000 \\
\hline Ecuador & 1,980 \\
\hline Indonesia & 1,950 \\
\hline Portugal & 1.920 \\
\hline Bolivia & 724 \\
\hline Honduras & 686 \\
\hline Cambodia & 569 \\
\hline Ethiopia & 537 \\
\hline Slovenia & 488 \\
\hline Tunisia & 470 \\
\hline Mongolia & 430 \\
\hline Slovakia & 419 \\
\hline Sudan & 419 \\
\hline Cameroon & 359 \\
\hline Guyana & 225 \\
\hline & \\
\hline & \\
\hline
\end{tabular}

Let us work to increase the true opportunities for undergraduates to do research, whatever the country.

\section{NOTICE TO SUBSCRIBERS}

AJUR's website will now provide full-color pdf [Adobe Acrobat] files of all articles. Our print version will continue with its current grayscale production. 